\title{
Cortisol does not mediate the suppressive effects of psychiatric morbidity on natural killer cell activity: a cross-sectional study of patients with early breast cancer
}

\author{
MALCOLM R. GARLAND*, ENA LAVELLE, DEREK DOHERTY, \\ LUCY GOLDEN-MASON, PATRICIA FITZPATRICK, ARNOLD HILL, \\ NOEL WALSH AND CLIONA O'FARRELLY
}

Education and Research Centre, St. Vincent's University Hospital, Elm Park, Dublih 4 and The Conway Institute, University College Dublin, Dublin 4, Ireland; Department of Immunology, National University of Ireland, Maynooth, Co. Kildare, Ireland; Department of Psychiatry, St. Vincent's University Hospital, Elm Park, Dublin 4, Ireland; Department of Public Health Medicine and Epidemiology, University College Dublin, Ireland; Department of Surgery, St. Vincent's University Hospital, Elm Park, Dublin 4, Ireland

\begin{abstract}
Background. There is evidence that depression impairs natural killer cell activity (NKA); this could have implications for anti-tumour immunity. Our aim was to examine the role of the hypothalamic-pituitary-adrenal (HPA) axis in suppressing NKA in a population of patients with early breast cancer, screened for depression. Secondary aims were to study the relationship between psychological, endocrine and immune variables and baseline tumour characteristics.

Metbod. A cross-sectional population of female patients $(n=55)$ with early breast cancer was sampled prior to primary surgery. Structured interview and psychometric instruments measured psychological distress. Flow cytometry was used to enumerate NK cells and lymphocytes were cryopreserved for use in a ${ }^{51} \mathrm{Cr}$-release assay, to estimate NKA. Midnight and three early morning saliva samples were collected to measure free cortisol levels. Tumour characteristics were obtained from hospital laboratory data.
\end{abstract}

Results. A high rate of psychological morbidity $(40 \%)$ was observed in the population. NKA was reduced in those with past or current psychiatric illness compared to those without ( $344 v .553 \mathrm{LU}_{20}$ and $455 v .569 \mathrm{LU}_{20}$ respectively, $p<0.05$ for both). Cortisol was not related to psychological status but was modestly positively correlated to NKA. A positive correlation was observed between the Fighting Spirit subscale of the Mental Adjustment to Cancer Scale and tumour size $(r=0.383$, $p=0.012$ ).

Conclusions. Our data support the evidence that psychological morbidity is associated with immune dysfunction; however, the most obvious candidate mediator of this effect, the HPA axis, does not appear responsible for this effect. Possible reasons for this are discussed.

\section{INTRODUCTION}

Rates of psychological morbidity in patients with cancer are high, particularly in those

- Address for correspondence: Malcolm R. Garland, Department of Psychiatry. Beaumont Hospital. Dublin 9. Ireland.

(Email: mgarland $a$ ireland com) recently diagnosed (McDaniel et al. 1995). There is evidence that depression may be associated with a poorer survival rate (Derogatis et al. 1979). If so, this may be due to illness behaviours, such as non-compliance and substance abuse, but it has also been suggested that the biological sequelae of depression, such as 
hypothalamic-pituitary-adrenal (HPA) axis dysregulation (Sephton et al. 2000) and impairment in immune function (Brittenden et al. 1996) may directly promote tumour growth. This intriguing and important possibility is supported by the well-documented immunosuppressive effects of glucocorticoids (GCs) and their anti-apoptotic effects on tumour cells (Mikosz et al. 2001). Natural killer (NK) cells are particularly susceptible to the effects of GCs (Garland et al. 2003), and as well as having important anti-tumour actions, are functionally impaired in depression (Zorrilla et al. 2001). Seminal work by Levy et al. $(1985,1987,1990)$ has demonstrated the independent association between natural killer cell activity (NKA) and both psychological and nodal status, at various stages of disease progression in breast cancer.

However, previous studies in populations with depression have generally failed to show a relationship between in vivo cortisol measures and reported decreases in either circulating NK cell numbers or NKA (Miller et al. 1991; Maes et al. 1992; Irwin et al. 1997), with only one study demonstrating a robust inverse NKAcortisol relationship (Irwin et al. 1988). Similar studies carried out in cancer populations have also been inconclusive. Lechin et al. (1990) found no NKA-plasma cortisol relationship in 50 advanced cancer patients and, more recently, neither did Sephton et al. (2000), publishing data examining the cortisol-NK cell relationship in an prospective population $(n=104)$ of metastatic breast cancer patients.

The above studies could be criticized on a number of methodological issues. Advanced cancer is not an ideal model to explore the cortisol-NK cell relationship due to the multiplicity of confounding factors associated with metastatic disease. Measuring single cortisol levels (all the above except Sephton et al. 2000 and Miller et al. 1991) imparts little information about the HPA axis. Also, the NK cell assay is prone to considerable day-to-day variation and efforts need to be made to control for this when testing at different time-points (Kane et al. 1996). None of the above studies appeared to have done this. Finally, only Miller et al. (1991) used a structured diagnostic interview to categorize psychological disorder. This study has addressed such issues.
The principal hypothesis was that in patients with primary breast cancer, psychological morbidity just prior to surgery was associated with impaired NK cell numbers and/or activity and mediating this effect was either an overactive and/or dysregulated HPA axis. Secondary hypotheses were that abnormal psychological/ endocrine/immune measures were associated with a poorer predicted disease outcome (as evidenced by tumour pathology). Several prospective studies have examined psychological distress at baseline and related it negatively to outcome (see Spiegel, 1997 for review). Altered cortisol rhythms have been reported in advanced cancer patients (Touitou et al. 1996) and have been linked to poor outcome (Sephton et al. 2000). Finally, the relatively few data on baseline NK cell parameters and prognosis in breast cancer (Brittenden et al. 1996) also warranted investigation.

\section{METHOD}

\section{Subjects}

The sample population comprised of 55 women (aged 28-74 years, mean 51.2 years) with stage I-IIIA mammary carcinoma, admitted under the surgical oncology team at St. Vincent's University Hospital, Dublin, for primary surgery. Patients were approached on the day of their admission, which was generally $24-48 \mathrm{~h}$ prior to surgery and informed written consent was obtained. The elapsed period from disclosure of diagnosis to admission was from 2 to 4 weeks. Standard demographic details were recorded, in addition to smoking status, alcohol consumption and menstrual/menopausal status. The presence of any past or family history of psychiatric or breast disorders was also noted. The following exclusion criteria were applied: bilateral carcinoma or carcinoma in situ; pre-operative chemotherapy or radiotherapy administration, pregnancy, co-morbid illness, acute or chronic, that could alter immune function; previous cancer or auto-immune disease; previous (within 6 weeks) or current treatment with sex hormones (including the oral contraceptive pill or hormone replacement therapy) or any immunologically active medication; psychotropic medication (apart from low-dose benzodiazepines); history of recent alcohol abuse (within 1 year) or psychosis. 
Oncological assessment and surgical procedure

Patients were assessed clinically and pathologically by means of standard international TNM (Tumour, Nodes, Metastasis) classification. All patients referred with a breast lump received fine-needle aspiration cytology and a core biopsy. Patients with positive histology were then referred for definitive surgery. This was either segmental (wide local excision, WLE) or modified radical mastectomy, with axillary node clearance to level III in both cases. Approximately 25 axillary nodes would then be sent for pathological examination. Pre-operatively, patients underwent staging procedures. All received a liver ultrasound, bone scan and chest $\mathrm{X}$-ray to rule out grossly evident metastatic disease. Carcino-embryonic antigen (CEA) and CEA 15.3 as well as oestrogen receptor status were measured. Where indicated, menopausal status was estimated by measuring gonadotrophin levels. Pathological examination in the hospital laboratory provided data on the size (the longest plane, in $\mathrm{cm}$ ) of the primary tumour, and the grade - from I-III depending on the degree of differentiation. The following tumour data were used in analyses:

(1) size $(\mathrm{cm})$;

(2) grade (I-III);

(3) axillary lymph nodes (number with tumour tissue present);

(4) percentage oestrogen receptor positivity;

(5) CEA and CEA 15.3 (IU/1).

\section{Laboratory methods - immunology}

Enumeration of $\mathrm{NK}$ cells

Venous blood was collected at 08:00 hours and processed immediately. Three-colour flow cytometric analysis, using a Becton Dickinson (Oxford, UK) FACScan flow cytometer was performed. Ten thousand events were acquired in each case. The percentage of CD $45+, C D 3-$, CD56 + (NK) cells were expressed in absolute numbers $\left(10^{3} / 1\right)$ by arithmetic conversion from a simultaneously obtained automated leucocyte count from the hospital haematology laboratory.

\section{Measurement of NKA}

Peripheral blood mononuclear cells (PBMCs) were prepared by standard density gradient centrifugation from $25 \mathrm{ml}$ heparinized blood. Cell pellets were frozen at $-80^{\circ} \mathrm{C}$ until required for testing in a standard 4-h chromium-51 $\left({ }^{51} \mathrm{Cr}\right)$ release NK cytotoxic assay (as described by Pross \& Maroun, 1984). The basis of this assay is counting of released radioactivity from target cells (K562 erythroleukaemia cells) upon exposure to lysis from NK ('effector') cells. Prior to use in the assay, cells were thawed and incubated overnight. Pooled PBMCs from a male volunteer were used as standard on each assay day, with subjects' values adjusted for variation in the mean of the standard, thus eliminating laboratory variability that is caused by dayto-day changes in target cell viability and the decline in $\gamma$-emission from ${ }^{5 t} \mathrm{Cr}$. Three effector to target $(\mathrm{E}: \mathrm{T})$ cell ratios (in triplicate) $-100: 1$, $50: 1$ and $25: 1$ were used. The percentage of specific radioactivity release for the three $E: T$ ratios was converted into lytic units (LU) set at the $20 \%$ level $\left(\mathrm{LU}_{20}\right)$ as described by Bryant et al. (1992), where $1 \mathrm{LU}$ is the number of effector cells required to kill $20 \%$ of the targets. This conversion into a single value eliminates the need for repeated statistical testing.

\section{Laboratory methods - salivary cortisol}

On the day of surgery, subjects provided one midnight and three early morning fasting samples (the latter separated by 15 -min intervals) of saliva by chewing on cotton wool pellets (Salivettes ${ }^{\mathbb{R}}$, Starsted, Ireland). Collection of the early morning samples commenced immediately upon awakening. Samples were refrigerated at $-20^{\circ} \mathrm{C}$ until analysed. The assay is an adaptation of a standard radio-immunoassay (Abbott Laboratories, Ireland). Intra- and interassay variability was less than $10 \%$. Two values were calculated from the raw data $(\mathrm{nmol} / \mathrm{l})$ : area under the curve (AUC) and standard deviation (S.D.). The former reflects mean concentrations over time and indicates the state of activation of the HPA axis, while the latter reflects variability, with low values indicating lack of diurnality and thus is a measure of dysregulation of the axis. Chronic stress/depression is associated with both increased basal activity and dysregulation of the HPA axis (Plotsky et al. 1998).

\section{Measurement of psychological morbidity}

For practical reasons psychometric testing took place between 5 and 7 days post-operatively. shortly prior to discharge, when patients were fully stable and mobilizing. As administered 
instruments measured either life traits or pervasive symptoms relating to the previous 4 weeks, it was felt there was not likely to be significant changes in such measures, from the immediate pre-operative period, when the biological samples were taken. The following instruments were administered:

The Schedule for Clinical Assessment in Neuropsychiatry (SCAN; WHO, 1998), Present State Examination, Version 10 (PSE-10)

A diagnostic computer programme (CATEGO) was used to process the data and scored according to the International Classification of Diseases (ICD)-10 (WHO, 1993) diagnostic criteria.

The Montgomery-Asberg Depression Rating Scale (MADRS; Montgomery \& Asberg, 1979)

The MADRS has been validated in the cancer population (Valente \& Saunders, 1997).

The Medical Outcomes Social Support Survey (SSS; Sherbourne \& Stewart, 1991)

This is a 20-item self-administered instrument and uses five-point answer scales. Four subscales exist:

(1) Positive social interaction;

(2) Emotional support;

(3) Affection:

(4) Tangible (i.e. practical) support,

Subscale scores are re-coded from one (worst) to 100 (best); a total score is calculated from the mean of the subscale scores. Only this latter score was used in statistical analysis.

\section{The Mental Adjustment to Cancer Scale} (MAC; Watson et al. 1988)

The MAC scale is a 40 -item scale, which has been developed to assess adjustments or responses to the diagnosis of cancer and is widely used as a measure of the coping styles employed by people with cancer. It incorporates the following five subscales:

(1) Fighting Spirit (16 items);

(2) Helplessness/Hopelessness (six items);

(3) Anxious Preoccupation (nine items);

(4) Fatalism (eight items);

(5) Avoidance (one item)

The scores from Fighting Spirit and Helplessness Hopelessness are amalgamated by subtracting
Table 1. Descriptive demographic data for the total population $(n=55)$

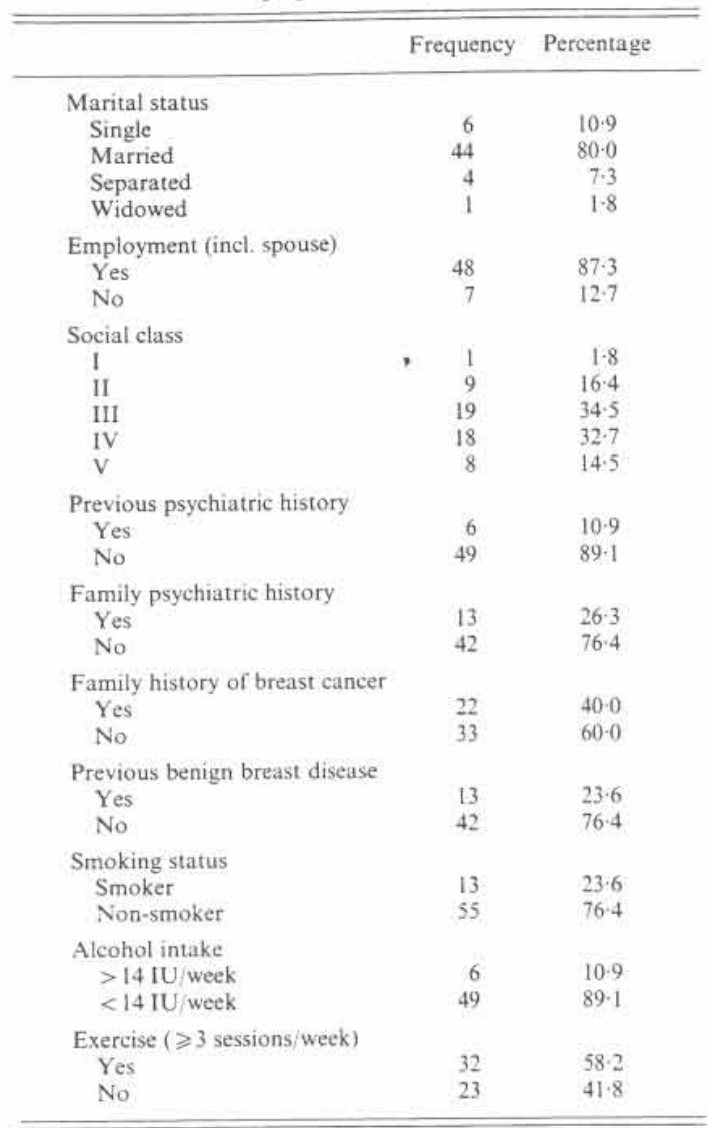

the latter from the former as they have been found to be psychometrically bipolar. As Avoidance is only one term, it does not constitute a subscale for scoring. High correlations are found between the Anxious Preoccupation subscale and the anxiety subscale of the Hospital Anxiety and Depression Scale (HADS; Zigmound \& Snaith, 1983) and also between the depression subscale of the latter and the Helplessness/Hopelessness subscale of the MAC (Watson et al. 1988). Of the three subscales used. Fighting Spirit is regarded as a 'positive' coping mechanism, the other two as 'negative'.

The SF-36 Version II (Jenkinson et al. 1999)

The SF-36 is a generic measure of health status, providing scores in eight areas of functioning 
Table 2. Clinical data $(n=55)$. Numbers in parentheses refer to percentage of patients in each category for categorical data and standard deviation from the mean for continuous data

\begin{tabular}{lc}
\hline \hline ICD-10 diagnosis & \\
Yes & $22(40)$ \\
No & $33(60)$ \\
Adjustment disorder (F43.2) & $12(21 \cdot 8)$ \\
Depression-mild (F32.0) & $5(9 \cdot 1)$ \\
Depression-moderate (F32.1) & $5(9 \cdot 1)$ \\
Tumour size (cm) & $2 \cdot 35(1 \cdot 48)$ \\
Tumour grade & $13(25 \cdot 5)$ \\
I & $21(41 \cdot 2)$ \\
II & $17(33 \cdot 3)$ \\
III & $4(7 \cdot 3)$ \\
Undetermined & \\
No. axillary nodes & $2 \cdot 51(6 \cdot 49)$ \\
Mean & $24(43 \cdot 6)$ \\
No. positive & $31(56 \cdot 4)$ \\
No. negative & \\
ER positive staining & $42(76 \cdot 4)$ \\
No. positive & $5(9 \cdot 1)$ \\
No. negative & $8(14 \cdot 5)$ \\
Undetermined & $6 \cdot 58(29 \cdot 4)$ \\
CEA (IU 1) & $21 \cdot 35(15 \cdot 2)$ \\
CEA 15.3 (IU/1) & \\
Menopausal & $34(61 \cdot 8)$ \\
Yes & $19(34 \cdot 5)$ \\
No & $2(3 \cdot 6)$ \\
Undetermined & \\
\hline \hline
\end{tabular}

and well-being: only the following three subscales were selected for statistical analysis.

(1) Role limitations due to emotional problems (three items);

(2) Mental health (five items);

(3) Energy/Vitality (four items).

The SF- 36 has been used satisfactorily in cancer patient populations (Anderson et al. 1996). The higher the scores on each subscale, the higher the positive health feelings.

\section{Statistical methods}

Data were analysed using SPSS for Windows, version 10.0 (SPSS Inc., 1999). Much of the laboratory data required transformation (either $\log _{10}$ or square root depending on the direction of skewness) to normalize distribution, due to the presence of a small number of outliers for most variables. The text in the Results section indicates if and how a variable was transformed. The associations between continuous variables were examined using two-tailed Pearson correlation co-efficients $(r)$. Comparison

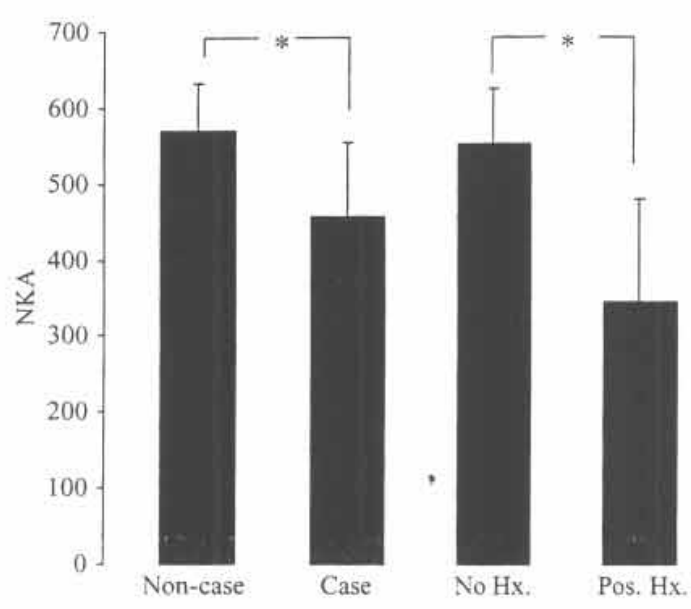

Fig. 1. Bar chart comparing the mean $(+5.8)$ of NKA in SCAN cases $(n=22)$ y, non-cases $(n=33)$ and patients with no previous psychiatric history (No Hx.) y. those with (Pos. Hx.). NKA is expressed as $\mathrm{LU}_{20}(455.3 v, 569 \cdot 5$. Case $v$. Non-case and 553.2 v. 344. No Hx. v. Pos. Hx.). * For log-transformed values of NKA $p<0.05$ for both comparisons (two-tailed independent samples $t$ test).

of means in two independent groups was performed using two-tailed independent $t$ tests, with multiple regression analysis. Where there were more than two categorical values for a variable (e.g. tumour grade), one-way analysis of variance (ANOVA), with post-hoc Bonferroni testing, was applied. Where two categorical measures were being compared, $\chi^{2}$ tests were used. Significance is indicated by a $p$ value of less than 0.05 .

\section{RESULTS}

\section{Descriptive data}

Summary categorical statistics for the patient population demographic data are presented in Table 1. Psychiatric, oncological, and menopausal data are detailed in Table 2. Those patients who were not menopausal were evenly divided with regard to phase of menstrual cycle (data not shown). Almost $60 \%$ were axillary node negative.

\section{Differences between SCAN cases and non-cases}

There were no significant differences in demographic (including smoking status) or tumourdata variables between SCAN cases and noncases. Lower NKA (but not NK cell numbers) 


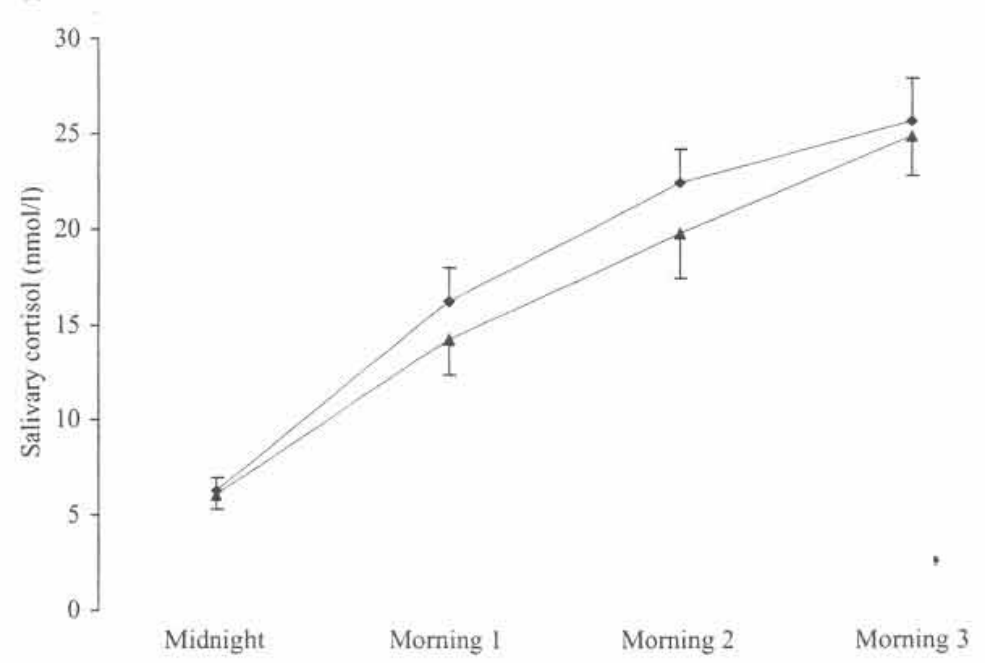

FiG. 2. Lineplot of mean salivary cortisol levels ( \pm S.E.M.) in SCAN non-cases ( $)$ and cases ( $\mathbf{A})$, for midnight and three early morning samples. The mean increase from midnight to early morning levels for the whole population was from $6 \cdot 1(0.45$ S.E.M. $)$ to $21.0(1: 2$ S.E.M. $) \mathrm{nmol} / \mathrm{I}(p=0.012)$.

was observed in both Axis I-positive cases and in those with a previous psychiatric history (Fig. 1), with no change in the significance after controlling for demographics, including smoking status. Non-smokers had significantly higher NKA (but not NK numbers) than smokers $\left(465.3\right.$ v. $\left.329 \cdot 1 \mathrm{LU}_{20}, p<0 \cdot 05\right)$. While menopausal patients had equivalent NKA they had higher NK cell numbers (195.1 v. 155.6, $p<0.05$ ). Data were too few to examine the effects of the menstrual cycle on immune parameters. No associations were found between immune data and other demographic variables, including age.

\section{Cortisol and NK cell parameters}

There was a robust increase from midnight to early morning cortisol levels (Fig. 2) indicating that subjects diurnal rhythms, were grossly intact. There was no difference in mean levels between cases and non-cases at any of the four measuring points. This was also the case after $\log$ transformation and comparison of AUC and S.D. values, indicating a lack of relationship between neither magnitude nor variability measures of the HPA axis and caseness. While there was no correlation between cortisol and enumerative NK data. AUC cortisol was (unexpectedly) positively related to NKA $(r=0.303, p<0.05)$.
Psychometric data: relationship to biological measures

All continuous psychometric data was normally distributed and did not require transformation. There were numerous strong intra- and interparameter correlates (Table 3), particularly between the MADRS and the SF-36 Quality of Life measure. Of note however, is the association of increasing Fighting Spirit (MAC) with depression scores. Also, there was no association between social support (SSS) and depression score.

As to the immune and tumour data, the only trend of interest was greater NKA in those with positive axillary nodes $[657.8( \pm 61.0$ S.E.M. $)$ in those with, $v .438 .8$ ( \pm 119.0 S.E.M.) in those without]; however statistical significance was not reached $(p=0.087)$. Finally, and of lesser importance to the central hypothesis, the relationship between the continuous psychometric data and the biological data was examined. There was no relationship between the former, including depression scores, and the endocrine data (data not shown). As regards the psychometric and tumour data, there was a positive relationship between tumour size and the Fighting Spirit subscale of the MAC Scale (Fig. 3), but no other significant inter-parameter relationship. Finally, for the immune data, total social support (SSS) and Energy/Vitality 
Table 3. Correlational data for continuous psychometric measures for the total population $(n=55)$. Pearson's $r$, with two-tailed significance $(p)$ beneath are displayed. Significant correlations are shown in bold

\begin{tabular}{|c|c|c|c|c|c|c|c|c|}
\hline & & $\begin{array}{c}\text { Social } \\
\text { support }\end{array}$ & $\begin{array}{l}\text { Fighting } \\
\text { Spirit }\end{array}$ & $\begin{array}{l}\text { Anxious } \\
\text { Preocc: }\end{array}$ & Fatalistic & $\begin{array}{c}\text { Role } \\
\text { limitation }\end{array}$ & $\begin{array}{l}\text { Mental } \\
\text { health }\end{array}$ & $\begin{array}{l}\text { Energy/ } \\
\text { Vitality }\end{array}$ \\
\hline \multirow[t]{2}{*}{ Depression" } & $r$ & -0.023 & 0.445 & $0 \cdot 326$ & $0-236$ & -0.288 & -0.629 & -0.439 \\
\hline & $p$ & 0.866 & 0.001 & 0.013 & $0-078$ & 0.030 & 0.000 & 0.001 \\
\hline \multirow{2}{*}{ Social support ${ }^{b}$} & $r$ & & -0.267 & -0.076 & -0.096 & 0.242 & 0.171 & $0 \cdot 269$ \\
\hline & $p$ & & 0.034 & 0.556 & 0.453 & 0.056 & 0.173 & 0.031 \\
\hline \multirow[t]{2}{*}{ Fighting Spirit ${ }^{c}$} & $r$ & & & 0.139 & $-0 \cdot 113$ & -0.286 & -0.397 & $-0 \cdot 268$ \\
\hline & $p$ & & & $0 \cdot 269$ & $0 \cdot 370$ & 0.026 & 0.001 & 0.032 \\
\hline \multirow[t]{2}{*}{ Anxious Preoce. ${ }^{\circ}$} & $r$ & & & & 0.253 & $-0 \cdot 322$ & $-0 \cdot 409$ & $-0 \cdot 164$ \\
\hline & $p$ & & & & 0.042 & 0.012 & 0.001 & 0.197 \\
\hline \multirow[t]{2}{*}{ Fatalistic ${ }^{\circ}$} & $r$ & & & & & -0.084 & -0.218 & -0.072 \\
\hline & $p$ & & & & & 0.520 & 0.083 & 0.574 \\
\hline \multirow[t]{2}{*}{ Role limitation ${ }^{4}$} & $r$ & & & & & & 0.470 , & 0.528 \\
\hline & $p$ & & & & & & 0.000 & 0.000 \\
\hline \multirow{2}{*}{ Mental health } & $r$ & & & & & & & 0.567 \\
\hline & $p$ & & & & & & & 0.000 \\
\hline
\end{tabular}

${ }^{2}$ MADRS; ${ }^{b}$ SSS; ${ }^{\text {MAC; }}{ }^{d}$ SF-36.

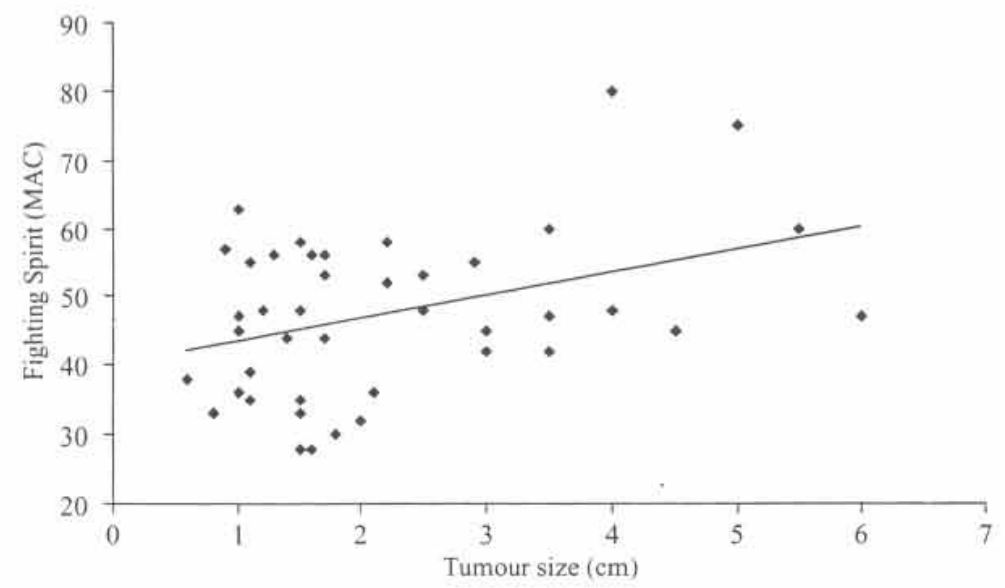

Fic. 3. Scatterplot (with linear regression line) of Fighting Spirit and tumour size. Pearson's $r=0 \cdot 383 . p=0 \cdot 012$.

(SF-36) were positively correlated with NK cell numbers $(r=0.301, p=0.014$, and $r=0.242$, $p=0 \cdot 048$ respectively) but not with NKA (data not shown).

\section{DISCUSSION}

In this cross-sectional study of 55 women with early breast cancer, rates of psychological morbidity comparable to the international literature were found. It is the largest such study to use a structured interview such as the SCAN. The central finding of this study was that while NK cell parameters were significantly lower in cases, this relationship was clearly not mediated by the HPA axis. In the complex jigsaw of Psychoneuro-immunology (PNI), pieces that fit at bench level (in this case, the well-documented in vitro suppression of $\mathrm{NK}$ cells by $\mathrm{GCs}$ ) often do not fit at the bedside.

Rates of psychological morbidity in early breast cancer

We report the rate of psychological morbidity (ICD-10) as $40 \%$, the majority being adjustment 
disorders. A previous study by Hall et al. (1999), used a shortened version of the PSE, which, in a population of 266 women with early breast cancer, identified $37.2 \%$ as being depressed, considerably higher than our own prevalence rates of $21 \cdot 8 \%$. They also identified $49 \cdot 6 \%$ of women as suffering from anxiety disorder (ICD-10, category F41), whereas we recognized none: all of the anxiety symptoms were sub-syndromal to F41, with many of these coded as adjustment disorder (F43.2). However the 'shortened' version of the PSE does not include the category of F43 (adjustment disorder) and this could have allowed for the over-diagnosis of depression and anxiety. Two other recent studies examine morbidity in early breast cancer (Ford et al. 1995; Aragona et al. 1997). Neither used a structured diagnostic interview. Aragona et al. (1997) found $55 \%$ of the cohort $(n=108)$ to be depressed. Ford et al. (1995) only found 7\% $(n=117)$ fulfilled HADS depression criteria.

Also of note are the high rates of previous history of psychiatric disorder $(10.9 \% v .6 .6 \%$ in a study by Morasso et al. 2001).

\section{HPA axis assessment: salivary cortisol}

As well as being non-invasive, the saliva method has the advantage of measuring not total, but free cortisol, as bound cortisol cannot enter saliva. Following the lead of established methodologies, the timing of the samples reflected both the awakening cortisol response and the diurnal pattern. The data was manipulated to best reflect either acute stress (corresponding to HPA activation), where mean, or AUC levels were used; or chronic stress (corresponding to HPA dysregulation), where one would examine for lack of variability/diurnality. We used standard deviation as a measure of variability. (For a review of salivary cortisol in psychiatric research see Kirschbaum \& Hellhammer, 1994.)

Without a control group, interpretation of our cortisol levels relative to normal populations must be cautious. However, using a similar laboratory methodology, Kirschbaum \& Hellhammer (1989) in a population of 662 healthy adults, found mean early morning cortisol to be 14.32 ( $\pm 9 \cdot 1$ S.D.) and a study by Edwards et al. (2001) reported a mean level of 10.53 ( \pm 3.0 S.D.) in a normal population of 40 . This is lower than our morning cortisol data, the mean of which was 20.97 ( \pm 11.7 S.D.), indicating our population, not surprisingly, had abnormally high levels of HPA axis activity. Nonetheless, in this study, cortisol measures in no way distinguished a psychologically distressed population from a non-distressed one, and this, considering its supposed ability to at least distinguish extreme forms of distress, prompts comment. Clearly, there were two forms of distress in operation with these patients. There was the 'acute' distress of anticipation of a major operation and its complications, and the 'chronic' distress of cancer diagnosis disclosure several weeks earlier. It was expected however, that the data would distingursh between these two forms of distress. Put simply, acutely distressed patients would have high AUC (magnitude) and chronically distressed patients would have low S.D. (variability) values of salivary cortisol. Perhaps the superimposition of an acute anticipatory stress on a chronic one simply created too much turbidity in the endocrine data to warrant reliable interpretation; none of the psychometric instruments used were designed to measure short-term (i.e. $24-48 \mathrm{~h}$ ) distress. If this is a fair criticism then one must ask when is the ideal time to sample an oncology population for these parameters? This was of course given consideration prior to the study, and just preoperatively was felt to be the optimum point. Post-operatively, one has to deal with differing anaesthetic, surgical and analgesic techniques and post-operative courses, all of which would confound the data. Any later and patients are in the chemotherapy and/or radiotherapy phases of treatment and the situation is similarly adverse to unconfounded data collection.

\section{Psycho-immune findings}

Our findings of low NKA in those with depression/adjustment disorder is in keeping with the literature (Zorrilla et al. 2001), although depression severity did not correlate with NK parameters. The positive association of the social support measure (in line with the findings of Levy et al. 1985, 1987, 1990) and the Energy/ Vitality subscale of the SF-36, with NK cell numbers is, however, evidence of an incremental relationship between psychological and immune factors. However the lack of association with cytotoxicity indicates a lack of consistency in the data. 


\section{The stress-cortisol-NK cell relationship}

The most salient laboratory finding of this study is that the impairment of NKA in patients with either a current or past psychiatric history is not related to cortisol levels. In fact, in keeping with the findings of Kronfol et al. (1997) in a normal population, we report a small, but significant, positive association between cortisol (AUC) and NKA. Despite there being a large literature demonstrating powerful NK cell inhibitory properties of cortisol in vitro (Garland et al. 2003), human in vivo studies have been equivocal (see Introduction). A human study that does report an association is that of Staurenghi et al. (1997), in a population of female patients with anorexia nervosa; they report a strong inverse correlation between 08:00 hours plasma cortisol levels and NKA. However, using a human surgical-stress model (laparotomy), Tonnesen et al. (1984) found no relationship in baseline or surgically induced changes in NKA and plasma cortisol. Bodner et al. (1998) found that the administration of dexamethasone to normal volunteers had no effect on NK cell distribution or activity. Moreover, Kronfol et al. (1997) reported that NK cell parameters displayed a diurnality that closely paralleled that of cortisol. Clearly, in vivo, there are factors such as the sympathetic nervous system and dynamic alterations in the $\mathrm{GC}$ receptor that modulate the immune response to circulating cortisol.

\section{Psycho-oncological findings}

Depression, categorical or continuous, was not related to tumour parameters. The most salient finding was the positive association with Fighting Spirit and tumour size. This is completely contrary to the landmark findings of Greer et al. (1979) who reported prolonged survival in those breast cancer patients with high baseline Fighting Spirit. However, contrary to the literature, we found a positive relationship between depression score and Fighting Spirit, indicating this measure may be sensitive to cultural influences.

\section{CONCLUSION}

This cross-sectional study once again confirms the high rates of psychological morbidity in patients with early breast cancer. Drawing firm conclusions from the neuroendocrine-immune perspective are more difficult, underpinning the difficulty in selecting medically ill populations at the optimum time, for sampling sensitive variables. Nonetheless there are sufficient positive findings to warrant follow-up of this cohort and future data will serve as an addition to the scant literature on the relationship of baseline psychological status to long-term survival. Similarly, the endocrine data of Sephton et al. (2000) needs replication and finally, no study has measured NK cell parameters at baseline in breast cancer and related them to survival.

\section{ACKNOWLEDGEMENTS'}

We thank the laboratory staff of the Haematology and Endocrinology Departments of St. Vincent's University Hospital for practical help. We acknowledge the useful discussions with Dr J. Crown and Professor T. Dinan at the setting-up stage. Dr G. Shakhar (Tel Aviv) provided help with lytic unit calculation. M.R.G. was supported by University College, Dublin as a Newman Scholar.

\section{REFERENCES}

Anderson. R. T., Aaronson, V. K., Bullinger, M. \& McBee, W. L. (1996). A review of the progress towards developing health related quality-of-life instruments for international clinical studies and outcomes research. Pharmacoeconomics 10. 336-355.

Aragona. M., Muscatello. M. R. \& Mesiti. M. (1997). Depressive mood disorders in patients with operable breast cancer. Journal of Experimental and Clinical Cancer Research 16, 111-118.

Bodner, G., Ho, A. \& Kreek, M. J. (1998). Effect of endogenous cortisol levels on natural killer cell activity in healthy humans. Brain Behavior Immunity 12. 285-296.

Brittenden, J., Heys, S. D., Ross, J. \& Eremin, O. (1996). Natural killer cells and cancer. Cancer 77, 1226-1243.

Bryant. J., Day, R., Whiteside, T. L. \& Herberman. R. B. (1992). Calculation of lytic units for the expression of cell-mediated cytotoxicity. Journal of Immunological Methods 146, 91-103.

Derogatis, L. R., Abeloff, M. D. \& Melisaratos, N. (1979). Psychological coping mechanisms and survival time in metastatic breast cancer. Journal of the American Medical Association 242. $1504-1508$.

Edwards, S., Evans, P., Hucklebridge, F. \& Clow, A. (2001). Association between time of awakening and diurnal cortisol secretory activity, Psychoneuroendocrinology 26, 613-622.

Ford, S., Lewis, S. \& Fallowfield, L. (1995). Psychological morbidity in newly referred patients with cancer. Journal of Psychosomatic Research 39. 193-202.

Garland, M.. Doherty, D., Golden-Mason, L.. Fitzpatrick. P., Walsh, N. \& O'Farrelly. C. (2003). Stress-related hormonal suppression of natural killer activity does not show menstrual cycle variations: implications for timing of surgery for breast cancer. Anticancer Research 23, 2531-2535.

Greer. S., Morris, T. \& Pettingale, K. W. (1979). Psychological response to breast cancer: effect on outcome. Lancet ii. 785-787.

Hall, A., A'Hern, R. \& Fallowfield, L. (1999). Are we using appropriate self-report questionnaires for detecting anxiety and 
depression in women with early breast cancer? European Journal of Cancer 35, 79-85.

Irwin, M.. Daniels, M., Risch, S. C., Bloom, E. \& Weiner, H. (1988). Plasma cortisol and natural killer cell activity during bereavement. Biological Psychiatry 24, 173-178.

Irwin, M., Hauger, R., Patterson, T. L., Semple, S., Ziegler, M. \& Grant, I. (1997). Alzheimer caregiver stress: basal natural killer cell activity, pituitary-adrenal cortical function, and sympathetic tone. Annals of Behavioral Medicine 19, 83-90.

Jenkinson. C., Stewart-Brown, S., Peterson, S. \& Paice, C. (1999). Assessment of the SF-36 Version II in the United Kingdom. Journal of Epidemiology and Community Medicine 53, 46-50.

Kane, K. L., Ashton. F. A., Schmitz, J. L. \& Folds, J. D. (1996). Determination of natural killer cell function by flow cytometry. Clinical and Diagnostic Laboratory Immunology 3, 295-300.

Kirschbaum, C. \& Hellhammer, D. H. (1989), Salivary cortisol in psychobiological research: an overview. Neuropsychobiology 22. $150-169$

Kirschbaum, C. \& Hellhammer, D. H. (1994). Salivary cortisol in psychoneuroendocrine research: recent developments and applications, Psychoneuroendocrinology 19, 313-333.

Kronfol, Z.. Nair, M. Zhang, Q., Hill, E. E. \& Brown, M. B. (1997) Circadian immune measures in healthy volunteers: relationship to hypothalamic-pituitary-adrenal axis hormones and sympathetic neurotransmitters. Psychosomatic Medicine 59, 42-50.

Levy, S. M.. Herberman, R. B., Maluish, A. M.. Schlien, B. \& Lippman, M. (1985). Prognostic risk assessment in primary breast cancer by behavioral and immunological parameters. Health Psychology 4, 99-113.

Levy, S. M., Herberman, R., Lippman, M. \& d'Angelo, T. (1987) Correlation of stress factors with sustained depression of natura killer cell activity and predicted prognosis in patients with breas cancer. Journal of Clinical Oncology 5. 348-353.

Levy, S. M., Herberman, R. B., Whiteside, T., Sanzo, K., Lee, J. \& Kirkwood, J. (1990). Perceived social support and tumor estrogen progesterone receptor status as predictors of natural killer cel activity in breast cancer patients. Psychosomatic Medicine 52 73-85

Lechin, F., van der Dijs, B., Vitelli-Florez, G., Lechin-Baez, S., Azocar, J., Cabrera, A., Lechin, A., Jara, H., Lechin, M., Gomez, F. et al. (1990). Psychoneuroendocrinological and immunological parameters in cancer patients: involvement of stress and deparameters in cancer patients: involvement of
pression. Psychoneuroendocrinology: 15, 435-451.

MeDaniel, J.S., Musselman, D. L., Porter, M. R., Reed, D. A. \& Nemeroff, C. B. (1995). Depression in patients with cancer. Diagnosis, biology, and treatment. Archives of General Psychiatry 52. 89-99.

Maes, M., Stevens, W., Peeters, D., DeClerck, L., Scharpe, S., Bridts, C., Schotte, C. \& Cosyns, P. (1992). A study on the blunted natural killer cell activity in severely depressed patients. Life Sciences 50 505-513

Mikosz, C. A., Brickley, D. R., Sharkey, M. S., Moran. T. W. \& Conzen, S. D. (2001) Glucocorticoid receptor-mediated protection from apoptosis is associated with induction of the serine threonine survival kinase gene, sgk-1.Journal of Biology and Chemistry 276, 16649-16654.

Miller, A. H., Asnis, G. M., Lackner, C., Halbreich, U. \& Norin, A. J. (1991). Depression. natural killer cell activity, and cortisol secretion. Biological Psychiatry 29.878-886.

Montgomery, S. A. \& Asberg, M. (1979). A new Depression scale designed to be sensitive to change. British Journal of Psychiatry 134, 382-389.

Morasso, G.. Costantini, M., Viterbori, P., Bonci, F., Del Mastro, L. Musso. M., Garrone, O. \& Venturini, M. (2001). Predicting mood disorders in breast cancer patients. European Journal of Cancer 37. 216-223

Plotsky, P. M., Owens, M. J. \& Nemeroff, C. B. (1998). Psychoneuroendocrinology of depression. Hypothalamic-pituitaryadrenal axis. Psychiatric Clinics of North America 21. 293-307.

Pross, H. F. \& Maroun, J. A. (1984). The standárdization of NK cell Pross, H. F. \& Maroun, J. A. (1984). The standardization of NK cell
assays for use in studies of biological response modifiers. Journal of Immunological Methods 68, 235-249.

Sephton, S. E., Sapolsky, R. M., Kraemer, H. C. \& Spiegel, D. (2000). Diurnal cortisol rhythm as a predictor of breast cancer survival. Journal of the National Cancer Institute 92, 994-1000.

Sherbourne, C. D. \& Stewart, A. L. (1991). The MOS social support survey. Social Science and Medicine 32.705-714.

Spiegel, D. (1997). Psychosocial aspects of breast cancer treatment. Seminars in Oncology 24 (suppl. 1), S1-36.

SPSS Inc. (1999). SPSS for Windows, Release 10.0.5. Standard Version. SPSS Inc.: Chicago. IL.

Tonnesen, E., Huttel, M. S., Christensen, N. J. \& Schmitz, O. (1984). Natural killer cell activity in patients undergoing upper abdominal surgery: relationship to the endocrine stress response. Acta Anaesthesiologia Scandanavica 28. 654-660.

Touitou, Y., Bogdan, A., Levi, F., Benavides, M. \& Auzeby, A. (1996) Disruption of the circadian patterns of serum cortisol in breast and ovarian cancer patients: relationships with tumour marker antigens. British Journal of Cancer 74, 1248-1252.

Valente, S. M. \& Saunders, J. M. (1997). Diagnosis and treatment of major depression among people with cancer. Cancer Nursing 20 $168-177$

Watson, M.. Greer, S., Young, J., Inayat. Q., Burgess, C. \& Robertson, B. (1988), Development of a questionnaire measure of adjustment to cancer: the MAC scale. Psychological Medicine 18. 203-209.

WHO (1993). The ICD-10 Classification of Menral and Behavioural Disorders Diagnostic Criteria for Research. World Health Organisation: Geneva.

WHO (1998), Schedules for Clinical Assessment in Neuropsychiarry. Version 2.1. World Health Organisation: Geneva.

Zigmound, A. \& Snaith, R. (1983). The Hospital anxiety and depression scale, Acta Psychiatrica Scandanavica 67, 361-370

Zorrilla, E. P., Luborsky, L., MeKay, J. R., Rosenthal, R., Houldin, A., Tax, A., McCorkle, R., Seligman, D. A. \& Schmidt, K. (2001). The relationship of depression and stressors to immunologicat assays: a meta-analytic review. Brain Behavior and Immunity 15 , 199-226 